\title{
Intramural platelet deposition in cerebral vasculopathy of systemic lupus erythematosus
}

\author{
D Ellison, K Gatter, A Heryet, M Esiri
}

\begin{abstract}
Aims: To test the hypothesis that fragments of platelet thrombi and vascular endothelium are incorporated into the walls of small cerebral vessels in systemic lupus erythematosus (SLE).

Methods: Six varied necropsy cases of central nervous system (CNS) SLE and 15 controls were studied. The controls were selected to represent a wide range of diseases in which the cerebral circulation is compromised. Tissue sections were stained by standard histochemical and immunocytochemical methods, the latter using antibodies to platelet membrane glycoprotein IIIa (CD61), and vascular endothelium (CD31).

Results: In four of six cases of CNS SLE characterised by small vessel hyalinisation and thickening, fragments of platelet membrane were found in the walls of small cortical and meningeal vessels. Similar findings were not evident in two other SLE cases that were characterised by relatively short clinical histories and an acute vasculitis. One control case of severe polyarteritis nodosa showed platelet fragment deposition in arteries larger than the vessels so affected in SLE.

Conclusions: Previous studies have suggested that neuropsychiatric symptoms in SLE may be related to repeated episodes of vasculitis in small cerebral vessels that are triggered by antiphospholipid antibodies. Concurrent thrombus formation might facilitate the incorporation of platelet fragments into small vessel walls. This process contributes to the thickening and irregularity of small vessels, a major feature of longstanding cases of CNS SLE.
\end{abstract}

(F Clin Pathol 1993;46:37-40)

Department of Pathology, John Radcliffe Hospital, Oxford

D Ellison

K Gatter

A Heryet

Department of

Neuropathology,

Radcliffe Infirmary,

Oxford

M Esiri

Correspondence to:

Dr D Ellison, Department of Pathology LE 56, South Academic Block,

Southampton General

Hospital, Tremona Road,

Southampton SO9 4XY.

Accepted for publication 13 July 1992 patients with SLE have been implicated in cerebrovascular damage. ${ }^{67}$ Transient inflammatory reactions involving such antibodies may, in combination with the action of anti- neuronal antibodies, underlie the fluctuating neuropsychiatric symptoms that occur during the course of SLE. ${ }^{89}$ The incorporation of platelet fragments from thrombi into vessel walls or the disruption of vascular endothelium might reasonably be expected during these inflammatory episodes, and may contribute to the cerebral vasculopathy in chronic cases. To investigate this possibility, we studied the vascular pathology in six cases of CNS SLE, using conventional histochemical methods and immunocytochemical methods with two antibodies. One of these detects platelet membranes (Y2/51: classified CD61), and the other labels vascular endothelium (JC70: classified CD31).

\section{Methods}

The clinical details of the six cases of CNS SLE that we examined are summarised in table 1. All six patients fulfilled the American Rheumatism Association's revised 1982 criteria for SLE. ${ }^{10}$ Fifteen control cases were selected for comparison. Three of these patients had normal brains, having died from myocardial infarction. The other cases were chosen because the cerebral vasculature had in some way been compromised. Two patients had had a single episode of cerebral infarction. Atherosclerosis and giant cell arteritis were the causes of multiple infarcts in two further cases. There were two patients who had polyarteritis nodosa with involvement of cerebral vessels. One of these had a particularly severe and widespread arteritis with fibrinoid necrosis. Five cases of CNS infection were included. Three were bacterial and two were fungal in origin. A reactive arteritis could be found in four of five of these cases; and small areas of infarction could be found in three of five cases. Fungal invasion of the vessels was seen in the example of Aspergillus meningitis.

Histological sections were cut from paraffin wax embedded, formalin fixed tissue. Routine histochemical methods (haematoxylin and eosin, cresyl violet and luxol fast blue, elastic van Gieson, Martius scarlet blue) were used. Immunocytochemistry was performed using the APAAP method as described previously. ${ }^{11}$ The primary antibodies were: Y2/51 which recognises CD61, platelet membrane glycoprotein $\mathrm{IIIa}^{12}$; and JC70 which recognises CD31, a membrane bound glycoprotein which occurs in vascular endothelium. ${ }^{13}$ Adjacent tissue sections were used for immunocytochemistry because of the capacity of JC70 occasionally to label platelets. ${ }^{13}$ A section to which serum, but not antibody, had been applied was included as a control.




Table 1 Details of patients with central nervous system SLE

\begin{tabular}{|c|c|c|c|c|}
\hline $\begin{array}{l}\text { Case } \\
\text { No }\end{array}$ & Age & Sex & $\begin{array}{l}\text { Duration of SLE } \\
\text { symptomis }\end{array}$ & CNS symiptoms/signs \\
\hline 1 & 41 & $\mathrm{~F}$ & 4 years & Transverse myelitis \\
\hline 2 & 47 & $\mathrm{~F}$ & 20 years & $\begin{array}{l}\text { Neuropsychiatric symptoms, limb paraesthesiae, } \\
\text { cranial nerve palsies }\end{array}$ \\
\hline 3 & 50 & $\mathrm{~F}$ & 9 years & $\begin{array}{l}\text { Neuropsychiatric symptoms, bilateral pyramidal } \\
\text { signs, cerebellar signs }\end{array}$ \\
\hline 4 & 53 & $\mathbf{M}$ & 1 year & Limb paraesthesiae, cerebellar signs \\
\hline 5 & 54 & $\mathrm{~F}$ & 17 years & Neuropsychiatric symptoms, epilepsy, tetraplegia \\
\hline 6 & 66 & $\mathrm{~F}$ & 6 years & Dementia, epilepsy, chorea \\
\hline
\end{tabular}

Table 2 Neuropathological features of six cases with CNS SLE

\begin{tabular}{|c|c|c|c|c|c|c|}
\hline Case No & 1 & 2 & 3 & 4 & 5 & 6 \\
\hline Brain weight $(\mathrm{g})$ & 1320 & 1220 & 1400 & 1580 & 970 & 1230 \\
\hline $\begin{array}{l}\text { Infarction* } \\
\text { Thrombosis }\end{array}$ & $\begin{array}{l}0 \\
\mathbf{o}\end{array}$ & $\begin{array}{l}+ \\
+\end{array}$ & + & + & $\begin{array}{l}+ \\
+\end{array}$ & $\stackrel{+}{+}$ \\
\hline Vasculitis $\nmid$ & o & + & + & + & o & o \\
\hline Small vessel vasculopathy $\ddagger$ & + & + & o & o & + & + \\
\hline Endothelial proliferation & o & + & o & o & + & + \\
\hline Perivascular haemorrhage & o & o & + & + & o & o \\
\hline Y2/51 staining & + & + & o & o & + & + \\
\hline
\end{tabular}

*macroinfarction or microinfarction

facute or subacute

thyaline thickening and eosinophilia. brains from patients with SLE was multifocal and ranged widely in size.

Vascular abnormalities were evident in all six SLE patients. A vasculopathy that manifests as hyaline thickening, eosinophilia, and sometimes endothelial proliferation of small vessels was seen in four of six cases (table 2). Deposits of material that were positively labelled by antibody Y2/51 (CD61) were found within the walls of a small proportion of thickened vessels in each of these cases (figs 2A and B). The deposits were nearly always found in meningeal and cortical vessels with a diameter of less than $100 \mu \mathrm{m}$, and up to 10 foci might be found in a typical section from the frontal pole. On adjacent sections from two of these four cases, JC70 (CD31) labelled material was very occasionally found at the same sites as the Y2/51 positive material (fig 3). However, other Y2/51 deposits in these cases were not matched by JC70 staining on adjacent tissue sections; and the latter antibody elsewhere delineated normal endothelium alone. Intramural fibrin was rarely found in the thickened small vessels of the four cases with vasculopathy (fig 4).

No intramural Y2/51 deposits were seen in two of six cases of SLE. A feature of both these cases was acute vasculitis. In one, an acute inflammatory infiltrate of polymorph neutrophils disrupted small vessels and spread out into the surrounding necrotic tissue. Thrombosis and perivascular haemorrhages were associated findings. Perivascular haemorrhage was a prominent feature in the other case where inflammatory cells congregated preferentially in venules. Vasculopathy was absent in both of these brains. When intramural Y2/51 positive material was associated with vasculitis

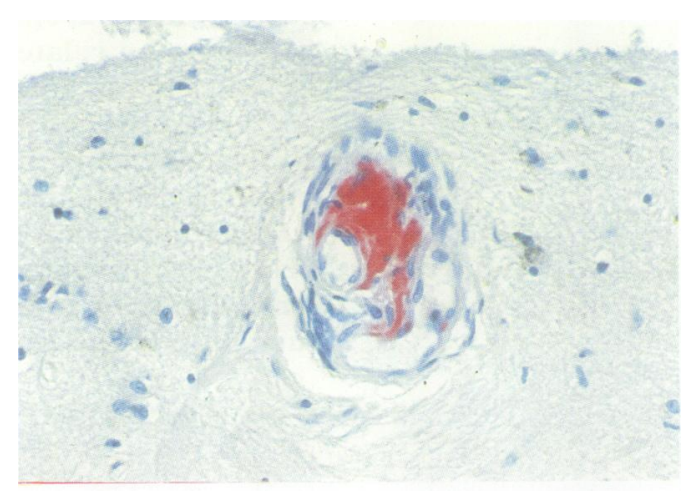

Figure 1 Acute vasculitis in the brain stem of case 4 (tables 1 and 2).

Polymorph neutrophils disrupt the vessel wall which shows fibrinoid necrosis, and spread into surrounding white matter (haematoxylin and eosin).

\section{Results}

This small series of patients with CNS neuropathological features (tables 1 and 2). oxample, one patient presented before death with his first CNS sympto while another had had the neuropsychiatric symptoms that are typical of SLE over 17 years. The range of pathology is exemplified by the findings in these two cases. The brain of the first patient weighed $1580 \mathrm{~g}$, which reflected the presence of oedema, petechial haemorrhages, and infarction secondary to acute vasculitis (fig 1); while the brain of the second patient weighed $970 \mathrm{~g}$, which reflected gross cerebral atrophy secondary to extensive laminar necrosis of the cortex. The laminar necrosis was present in a distribution that suggested numerous foci of ischaemia; and cystic infarction was present in many other areas of this brain. Recent or old infarction in five of six

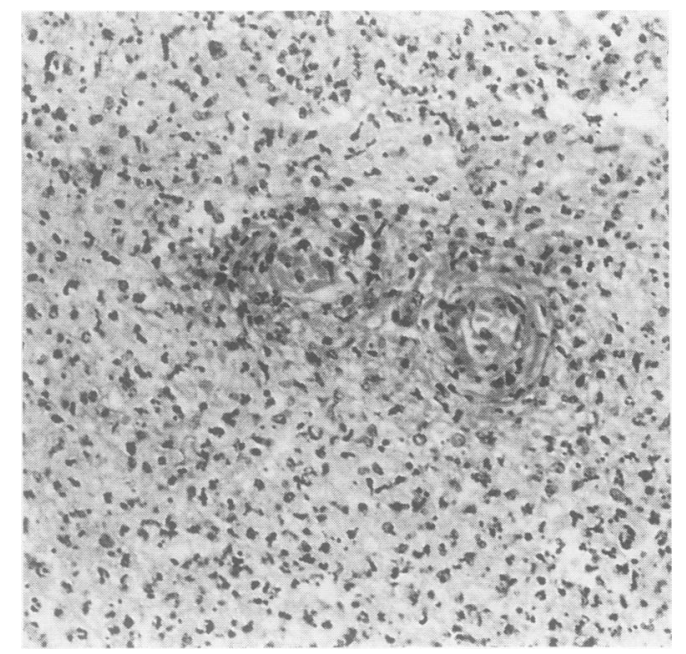

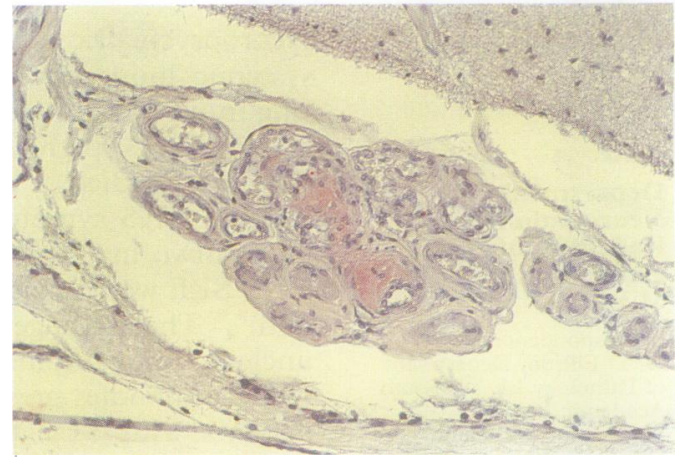

Figures $2 A, B$ Intramural Y2/51 positive material (red) is present in cortical $(A)$ and meningeal $(B)$ vessels from two patients with CNS SLE (haematoxylin counterstain). 


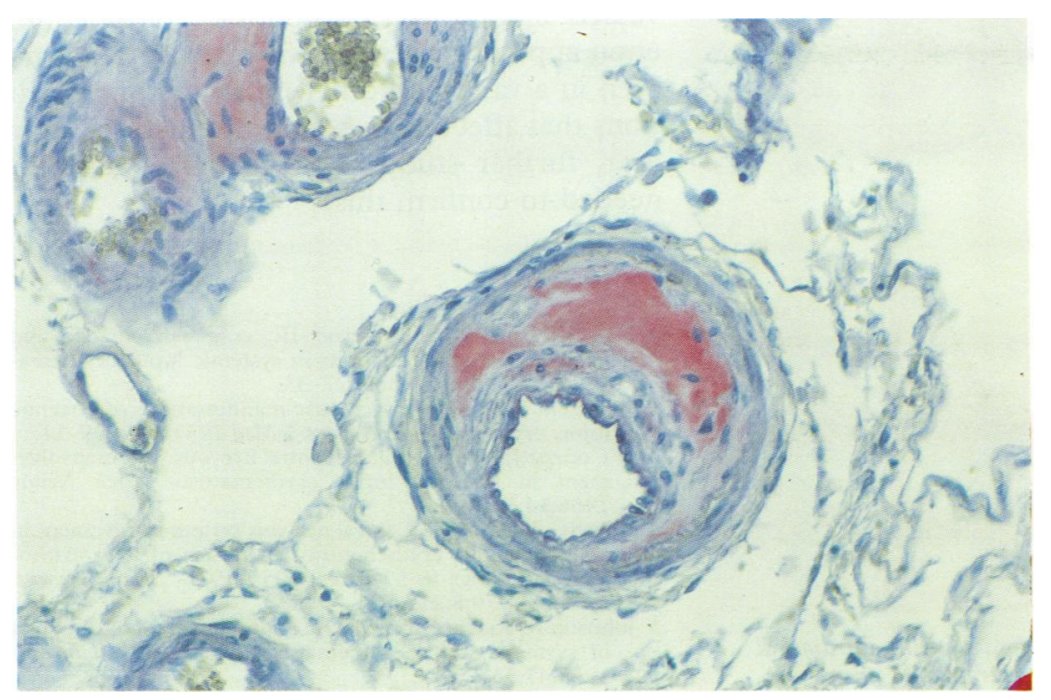

Figure 3 Intramural material and vascular endothelium labelled with $\mathfrak{F C 7 0 . T h i s ~}$ antibody is cross-reacting with platelet derived material which labelled identically with Y2/51 in the adjacent section (haematoxylin counterstain).

in a separate patient (case 2 ; tables 1 and 2), the inflammatory infiltrate was patchy and consisted of lymphocytes and macrophages, rather than polymorph neutrophils; and a vasculopathy was present (fig 5 ).

No intramural deposits of Y2/51 or JC70 labelled material were found in the non-SLE cases, with one exception. A few medium sized vessels ( $>100 \mu \mathrm{m}$ in diameter) in the case of polyarteritis nodosa with fibrinoid necrosis contained intramural Y2/51 (fig 6). Intravascular thrombi and vascular endothelium were consistently labelled with $\mathrm{Y} 2 / 51$ and JC70, respectively, throughout the series of brains.

\section{Figure 4 Fibrin} deposition in the wall of a cortical vessel which is disrupted and surrounded by an inflammatory infiltrate (MSB).

Figure 5 Subacute vasculitis in small vessels showing marked thickening, and adventitial thickening, and intramural lymphocytes (haematoxylin and eosin).

\section{Discussion}

The six cases of CNS SLE are characterised by a wide spectrum of neuropathological features. This spectrum encompasses all the cerebrovascular pathology reported in two studies of the brains of patients with CNS SLE. ${ }^{45}$ These studies concluded that while acute vasculitis was rare in a large postmortem series of SLE cases, "vasculopathy" was a common finding. Vasculopathy includes hyaline thickening and eosinophilia of the vessel wall, fibrinoid degeneration, and endothelial proliferation. It may be accompanied by thrombosis.

Vasculopathy of this type was seen in four of six cases of SLE in the present study. Intramural platelet fragments detected by antibody Y2/51 were seen in small ( $<100 \mu$ m diameter), thickened vessels in the same four cases. We could find no intramural deposits of Y2/51 material in the two other SLE cases. An acute and devastating brain stem and cerebellar vasculitis was the first CNS lesion for one of these patients (case 4; tables 1 and 2), occurring one year after his first symptoms of SLE. No feature of a cerebral vasculopathy was found in this brain or that of the other patient (case 3 ; tables 1, and 2) who had had the symptoms of CNS SLE for five months before her death. These patients did not have a long history of CNS symptoms in contrast to the four with vasculopathy. Vasculopathy may be the hallmark of repeated attacks of CNS SLE over several years, and the incorporation of platelet membranes into the walls of small vessels may be an epiphenomenon of the attacks.

Arteriosclerosis comprises elements of the vasculopathy described above and commonly affects the cerebral vasculature. Hyaline thickening of small vessels is particularly evident in hypertensive patients. The incorporation of platelet fragments into arteriosclerotic small vessels was not seen in the brains of our control patients.

The incorporation of platelets into atherosclerotic plaques is well recognised, ${ }^{14}$ but atherosclerosis is a disease of large and medium sized arteries, rather than the small vessels affected in CNS SLE.

Cerebrovascular abnormalities dominate the neuropathology of SLE, but their precise association with symptomatology has yet to be determined. ${ }^{2}$ Exacerbations of CNS SLE correlate with reduced cerebral blood flow on single photon emission tomógraphy, ${ }^{15}$ and with complement activation. ${ }^{16}$ Immune complex deposition and vasculitis associated with complement activation can be focal and short-lived, and may account for fluctuating neuropsychiatric symptoms. Antineuronal antibodies probably contribute to these phenomena, gaining access to the brain during a temporary breakdown in the blood-brain barrier consequent on vasculitis. ${ }^{89}$ Both the vasculitis itself and any residual vessel damage may produce infarction and permanent symptomatology.

Antiphospholipid antibodies are associated with cerebral infarction in patients with SLE, ${ }^{617}$ and some of these have an anti- 


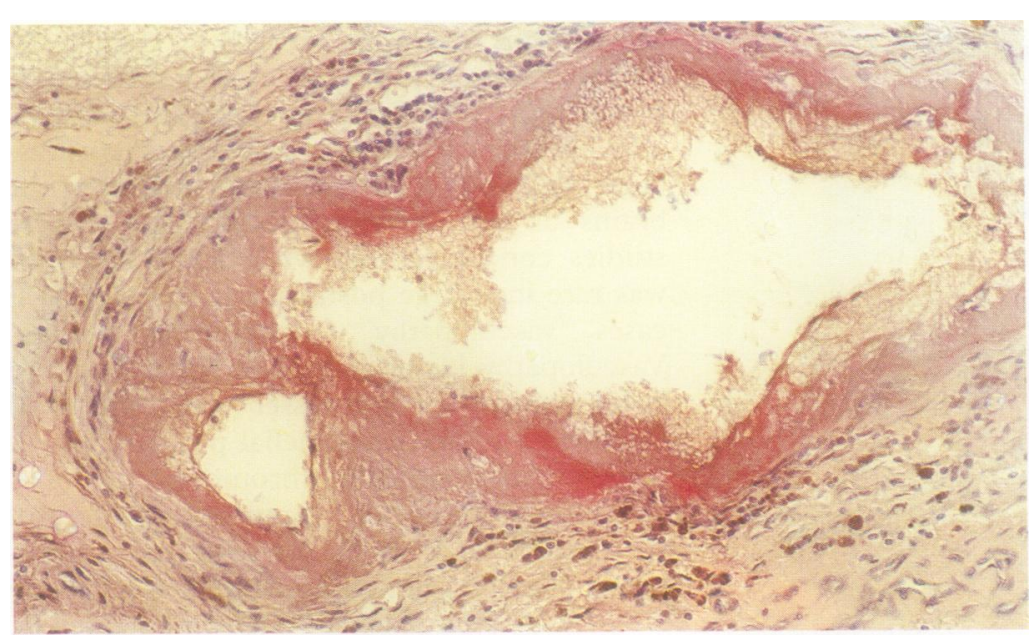

Figure 6 Medium sized vessel from a case of polyarteritis nodosa showing intramural deposition of $Y 2 / 51$ positive material and haemosiderin laden macrophages in the surrounding tissue (haematoxylin counterstain).

endothelial action which may be associated with hypofibrinolysis. ${ }^{18}$ Such autoimmune activity could be the setting for thrombus formation and transient focal ischaemia in small vessels. Focal ischaemia followed by reperfusion predisposes to platelet deposition along small vessels, ${ }^{19}$ and may facilitate the intramural incorporation of platelet derived material during resolution of the inflammatory process.

Antiphospholipid antibodies are also associated with Libman-Sacks endocarditis, and emboli from cardiac lesions may contribute to the cerebrovascular lesions. ${ }^{17}$ However, both the referenced pathological studies ${ }^{45}$ found no consistent association between cerebral lesions and endocarditis. Libman-Sacks endocarditis was found in only one of our cases (case 6; tables 1 and 2). However, its absence at necropsy in the other SLE patients does not discount a similar process at some time during their histories of CNS symptoms.

In conclusion, we have shown the intramural deposition of platelet derived material in small cerebral vessels in four cases of CNS SLE. This was not evident in two other cases where a history of CNS SLE was relatively short, and where thickening and eosinophilia of small vessels were not found. Intramural platelet deposition in association with vasculopathy may follow repeated episodes of inflammation. A similar process may occur in slightly larger vessels in polyarteritis nodosa. The phenomenon appears rather specific because it was not seen in a variety of other inflammatory conditions that affect the cerebral vasculature. However, further study of more cases would be needed to confirm this.

1 Lim L, Ron MA, Ormerod IE, et al. Psychiatric and neurological manifestations in systemic lupus erythematosus. OfMed 1988;66:27-38.

2 Bluestein HG. Neuropsychiatric manifestations of systemic lupus erythematosus. $N$ Engl f Med 1987;317:309-11.

3 O'Connor JF, Musher DM. Central nervous system involvement in systemic lupus erythematosus. Arch Neurol 1966;14:157-64

4 Ellis SG, Verity MA. Central nervous system involvement in systemic lupus erythematosus: a review of neuropathological findings in 57 cases, 1955-1977. Semin Arthritis Rheum 1979;8:212-21.

5 Johnson R, Richardson EP. The neurological manifestations of systemic lupus erythematosus. Medicine (Baltimore) 1968;47:337-69.

6 Asherson RA, Khamashta MA, Gil A, et al. Cerebrovascular disease and antiphospholipid antibodies in systemic lupus erythematosus, lupus-like disease, and the primary phoserythematosus, lupus-like disease, and the primary

7 Cines DB, Lyss AP, Reeber M, Bina M, DeHoratius RJ. Presence of complement-fixing anti-endothelial cell antiPresence of complement-fixing anti-endothelial cell antibodies in system

8 Bluestein HG, Williams GW, Steinberg AD. Cerebrospinal fluid antibodies to neuronal cells: association with neuropsychiatric manifestations of systemic lupus erythematosus. $A m$ F Med 1981;70:240-6.

9 How A, Dent PB, Liao S-K, Denburg JA. Antineuronal antibodies in neuropsychiatric systemic lupus erythematosus. Arthritis Rheum 1985;28:789-95.

10 Tan EM, Cohen AS, Fries JF, et al. The 1982 revised criteria for the classification of systemic lupus erythematosus. for the classification of systemic
Arthritis Rheum 1982;25:1271-7.

11 Cordell JL, Falini B, Erber WN, et al. Immunoenzymatic labelling of monoclonal antibodies using immune complexes of alkaline phosphatase and monoclonal antialkaline phosphatase (APAAP complexes). $f$ Histochem Cytochem 1984;32:219-29.

12 Gatter KC, Cordell JL, Turley $\mathrm{H}$, et al. The immunohistochemical detection of platelets, megakaryocytes, and thrombi in routinely processed specimens. Histopathology 1988;13:257-67.

13 Parums DV, Cordell JL, Micklem K, Heryet AR, Gatter KC, Mason DY. JC70: a new monoclonal antibody that detects vascular endothelium associated antigen in routinely processed sections. $\mathcal{F}$ Clin Pathol 1990;43:752-7.

14 Fuster V, Badimon L, Badimon JJ, Cheseboro JH. The pathogenesis of coronary artery disease and the acute coronary artery syndromes. $N$ Engl $f \mathrm{Med} 1992$; 326:242-50.

15 Kushner MJ, Tobin M, Fazekas F, et al. Cerebral blood flow variations in CNS lupus. Neurology 1990;40:99-102.

16 Hopkins P, Belmont HM, Buyon J, Philips M, Weissmann $G$, Abramson SB. Increased levels of plasma anaphylotoxins in systemic lupus erythematosus predict flares of the disease and may elicit vascular injury in cerebritis. Arthritis Rheum 1988;31:632-41.

17 Asherson RA, Lubbe WF. Cerebral and valve lesions in SLE: association with antiphospholipid antibodies. $f$ Rheumatol 1988;15:539-43.

18 Angles-Cano E. Endothelial damage and hypofibrinolysis in systemic lupus erythematosus. Thromb Haemostas 1989;61:322.

19 Jafar JJ, Menoni R, Feinberg H, LeBreton G, Crowell RM. Selective platelet deposition during focal cerebral ischaemia in cats. Stroke 1989;20:664-7. 